\title{
Nonsingular PNG-Based Impact Time Control Guidance with Lower Dependence on Time-to-Go Estimate
}

\author{
Sijiang Chang $\mathbb{D}^{1}$ and Shengfu Chen $\mathbb{D}^{2}$ \\ ${ }^{1}$ Nanjing University of Science and Technology, 210094 Nanjing, China \\ ${ }^{2}$ The 20th Research Institute of China Electronics Technology Group Corporation, 710068 Xi'an, China \\ Correspondence should be addressed to Sijiang Chang; ballistics@126.com
}

Received 19 April 2019; Accepted 8 November 2019; Published 25 November 2019

Academic Editor: Antonio Viviani

Copyright (c) 2019 Sijiang Chang and Shengfu Chen. This is an open access article distributed under the Creative Commons Attribution License, which permits unrestricted use, distribution, and reproduction in any medium, provided the original work is properly cited.

\begin{abstract}
In a bid to take advantage of natural characteristics of the proportional navigation guidance (PNG) in practical engineering, the PNG-based impact time control guidance (ITCG) continues to be a popular alternative for achieving the desired impact time of a missile. For most such ITCG, the performance is dependent on the accuracy of the time-to-go estimation. Along the lines of the development of PNG-based ITCG in earlier studies, a nonsingular ITCG is proposed on the basis of nonlinear formulations. It is demonstrated that, by theoretical analysis and numerical simulation, this proposed ITCG is shown to be advantageous in certain circumstances. By deriving a novel additional acceleration command, the proposed law is of lower dependence on timeto-go estimate and is capable of eliminating some singularities, leading to wider adjustable range of the desired impact time and better adaptability to more conditions. This research is expected to be supplementary to those presented in the current research literature.
\end{abstract}

\section{Introduction}

In recent years, the impact time control guidance (ITCG) law has been widely studied to meet various requirements of modern battlefield missions and tasks. Because the ITCG enables a missile or a group of missiles, to hit the target at a desired impact time, this guidance law can be used to address the issues associated with salvo attack and cooperative attack. The first appearance of the ITCG was in [1], where the authors successfully derived a linear-formulation-based closed form solution comprising the proportional navigation guidance (PNG) law and the feedback of the impact time error. Since then, a raft of ITCG laws in various forms have been developed.

Moreover [1], a number of ITCG laws presented in the current research literature [2-17] are also dependent on the time-to-go estimation, although they may be designed based on different theories or techniques. For instance, the studies presented in [1-10] were performed in terms of the principle of PNG, among which several widely used formulas for estimating the time-to-go are also derived in the framework of
PNG. A sliding-mode-based ITCG law was derived in [11] with a simple time-to-go estimate that uses the range divided by the closing speed. Three specific ITCG laws based on sliding mode technique were proposed in [12-14], using the same time-to-go approximation proposed in [2]. The Lyapunov-based ITCG law as presented in [15] uses the time-to-go formula derived in [1]. The concept of polynomial trajectory guidance was developed in $[16,17]$ to address the impact time control. In [16], the time-to-go was obtained by solving the nonlinear equations of motion for missiles, while Ref. [17] presents an improved calculation of time-to-go via deriving a closed-form solution of arc length. It has been demonstrated by the results of the above studies that the performance of the ITCG that requires time-to-go is significantly affected by the accuracy of the time-to-go estimation.

To avoid considerable effort to time-to-go, the ITCG law without using time-to-go has been developed in recent studies [18-31]. Tekin et al. [18-22] proposed a series of notable guidance laws under various conditions using polynomial shaping of the look angle and range and the feedback 
linearization technique. Gutman [23] discussed a novel guidance law for impact time control using the concept of the zero-effort miss vector. Saleem and Ratnoo [24] derived a closed-form expression for the impact time and then achieved the desired impact time. In [25], an ITCG law in the form of PNG was proposed by calculating the timevarying navigation gain instead of estimating time-to-go. Several sliding-mode-control-based ITCG laws that do not involve time-to-go were designed in [26-28]. A Lyapunovbased ITCG with an exact solution for impact time instead of time-to-go estimate was proposed in [29]. The optimal control theory can also be used to achieve the impact time control without using time-to-go estimation, as presented in $[30,31]$.

It can be seen from the aforementioned references that most proportional-navigation-based ITCG laws require the time-to-go estimation and the time-to-go estimation derived in the framework of PNG can also be incorporated into the ITCG law that depends on other theories or techniques rather than PNG. For the proportional-navigation-based ITCG laws, because these guidance laws closely approach the PNG as the impact time error goes to zero, the trajectory eventually evolves into a PNG trajectory regardless of the design method. From information requirement perspective, the ITCG law that does not involve time-to-go estimation seems to be superior over that needs time-to-go since it eliminates the effect of time-to-go estimation error on the guidance performance. From practical point of view, to make use of excellent characteristics of PNG that has already been widely validated in practical engineering over the past several decades, the proportional-navigation-based ITCG law is worthy of being developed in certain aspects such as how to reduce its dependence on the accuracy of the timeto-go estimation.

The main motivation for the results of this paper came from [6], where the authors derived a novel ITCG with generalized proportional navigation based on nonlinear formulations and provided a quite accurate higher-order time-togo estimate having significant effect on the ITCG performance. As stated in [6], despite its rigorous form and effectiveness, the ITCG of [6] still has an operational limitation in that the desired impact time must be larger than the impact time of the PNG trajectory. This effort reported here is along the lines of the study on ITCG in [6] and develops a nonsingular proportional-navigation-based ITCG with lower dependence on the time-to-go estimation. The proposed ITCG allows the desired impact time to be larger or smaller than the impact time of the PNG trajectory and is capable of eliminating the singularity driven by inaccurate time-to-go estimate, resulting in broader controllable range of desired impact time and augmented adaptability to more conditions. In addition, this study shows that by using a switching logic pertinent to the additional acceleration command, the proposed ITCG can address the field-ofview constraint efficiently and still be effective for some extreme conditions.

The organization of this paper is as follows. In Section 2, a brief description of this problem is given. Section 3 covers the derivation of the nonsingular proportional-navigation- based ITCG law with the seeker's field-of-view limit in detail. Significant characteristics of the presented ITCG such as the convergence, the dependence on time-to-go estimate, and the controllable range of the desired impact time are theoretically analyzed in Section 4. A numerical simulation study is presented in Section 5, and Section 6 presents concluding remarks.

\section{Problem Statement}

The problem studied in this paper is parallel to that presented elsewhere [6]. For the sake of completeness, this section is aimed at introducing the necessary notations and equations used in the subsequent sections. We consider a twodimensional missile-target engagement scenario for a stationary target with the seeker's field-of-view constraint, which means that this problem can be described as a scalar system. In the interest of convenience, it is assumed that the speed of the missile $V$ is constant and the autopilot lag is neglected. The engagement geometry is shown in Figure 1, where $R$ is the distance between the missile and the target; $\gamma$ and $\theta$ represent the flight path angle and the line-of-sight angle, respectively; $\phi, \phi_{0}$, and $\phi_{\max }$ denote the heading error, the initial heading error, and the boundary of the field-of-view angle, respectively; $a_{m}$ represents the acceleration command, and the coordinate $(x, y)$ can be used to describe the trajectory of the missile.

The governing differential equations are expressed as follows:

$$
\begin{aligned}
& \dot{R}=-V \cos \phi, \\
& \dot{\theta}=-\frac{V \sin \phi}{R}, \\
& \dot{\gamma}=\frac{a_{m}}{V}, \\
& \phi=\gamma-\theta,
\end{aligned}
$$

where the dot over the variant denotes the first derivative with respect to the time $t$.

Like in [6], the total acceleration command $a_{m}$ consists of two parts, i.e.,

$$
a_{m}=a_{b}+a_{\xi}
$$

where $a_{b}$ is a feedback acceleration command that is used to minimize the missed distance as much as possible and $a_{\xi}$ is an additional acceleration command that is responsible for controlling the impact time.

The heading error rate can be written as follows:

$$
\dot{\phi}=\dot{\gamma}-\dot{\theta}=\frac{a_{b}+a_{\xi}}{V}+\frac{V \sin \phi}{R} .
$$

To accomplish the engagement, the heading error must satisfy the condition $|\phi| \leq \phi_{\max }$ over the total trajectory. For convenience, it is assumed that $\left|\phi_{\max }\right|<\pi$ and $\phi_{\max } \neq \pm$ $\pi / 2$. By using Equations (1), (2), (3), (4), (5) and (6) and 


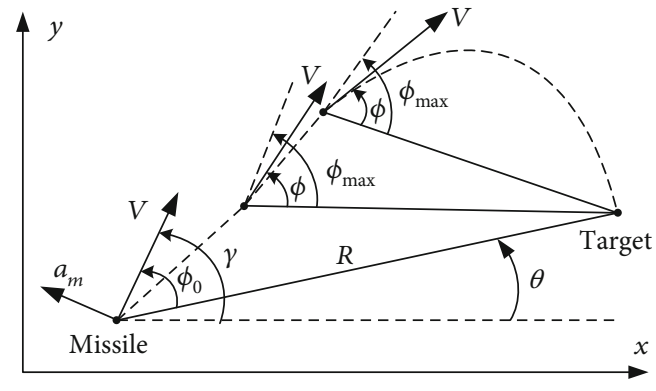

FIGURE 1: Engagement geometry for stationary target with field-ofview constraint.

introducing a variable $\eta=\sin \phi$, the following Equations (7) and (8) can be obtained:

$$
\frac{d \eta}{d R}+\frac{\eta}{R}=-\frac{a_{b}+a_{\xi}}{V^{2}}, \quad \eta \in(-1,1) .
$$

The boundary condition of Equation (7) is $\eta\left(R_{0}\right)=$ $\eta_{0}=\sin \phi_{0}$ and $\eta(0)=\eta_{f}=0$, where $R_{0}$ is the initial distance between the missile and the target. Note that the subscripts " 0 " and " $f$ " denote the initial and final states, respectively.

$$
\frac{d t}{d R}=-\frac{1}{V \sqrt{1-\eta^{2}}} .
$$

The boundary condition of $(8)$ is $t\left(R_{0}\right)=0$ and $t\left(R_{f}\right)=t_{f}$, where $R_{f}$ is the final distance between the missile and the target and $t_{f}$ is the desired impact time.

Therefore, the total acceleration command $\left(a_{b}+a_{\xi}\right)$ depending on nonlinear Equations (7) and (8) is the desired command that can effectively control the impact time of the missile.

\section{Derivation of Impact Time Control Guidance Law}

The derivation of the proposed ITCG starts from transforming the problem of solving nonlinear Equations (7) and (8) into an optimal control problem, which is parallel to the approach used in [6]. For completeness, this section intends to briefly restate the derivation which is similar to [6] and focus on the finally obtained results which differ from that of [6] in essence.

Assumed that the additional command $a_{\xi}$ is constant, the control effort to be minimized subject to Equation (7) can be chosen as follows:

$$
J=\frac{1}{2} \int_{0}^{R_{0}} \frac{a_{b}^{2}}{R^{m}} d R,
$$

where $m$ is an integer.

To solve the optimal control problem comprising Equations (7) and (9), a Hamilton function $H$ is constructed as follows:

$$
H=\frac{a_{b}^{2}}{2 R^{m}}+\lambda_{\eta}\left(-\frac{a_{b}+a_{\xi}}{V^{2}}-\frac{\eta}{R}\right)
$$

where $\lambda_{\eta}$ is a Lagrange multiplier to be determined.

According to the minimum principle in the optimal control theory, it yields

$$
\begin{aligned}
& \frac{d \lambda_{\eta}}{d R}=-\frac{\partial H}{\partial \eta}=\frac{\lambda_{\eta}}{R}, \\
& \frac{\partial H}{\partial a_{b}}=\frac{a_{b}}{R^{m}}-\frac{\lambda_{\eta}}{V^{2}}=0 .
\end{aligned}
$$

Combining Equations (11) and (12) with Equation (7) and then performing a series of algebraic operations with the boundary conditions $\eta_{f}=0$ and $R_{f}=0$, the mathematical relation between $a_{b}$ and $a_{\xi}$ is obtained as follows:

$$
a_{b}=-N \frac{\eta}{R} V^{2}-\frac{N}{2} a_{\xi}
$$

where the constant $N$ is defined as $N=3+m$ and $m>-1$. Obviously, this equation becomes the PNG as $a_{\xi}$ converges to zero.

Substituting Equation (13) into Equations (7) and (8) and integrating, a quadratic equation with respect to the additional command $a_{\xi}$ can be obtained as follows:

$-\frac{(N-2)^{2} R^{3}}{12(N+1)(2 N-1)}\left(\frac{a_{\xi}}{V^{2}}\right)^{2}+\frac{(N-2) R^{2} \eta}{2(N+1)(2 N-1)}\left(\frac{a_{\xi}}{V^{2}}\right)+\xi_{T}=0$,

where the impact time error is denoted as $\xi_{T}=t_{f d}-t_{\mathrm{goPN}}$, in which $t_{\mathrm{goPN}}$ and $t_{f d}$ represent the estimated time-to-go and the real time-to-go, respectively.

By solving the quadratic Equation (14) directly and making some corrections, a novel closed form solution is given as follows:

$$
\begin{aligned}
a_{\xi}= & \frac{3 V^{2}}{N-2} \frac{\eta}{R}-h\left(\xi_{T}\right) \\
& \cdot \delta(\eta) \sqrt{\left(\frac{3 V^{2}}{N-2} \cdot \frac{\eta}{R}\right)^{2}+\frac{12(N+1)(2 N-1) V^{5}}{(N-2)^{2} R^{3}} \cdot\left|\xi_{T}\right|},
\end{aligned}
$$

where $\delta(\cdot)$ is a sign function defined as follows:

$$
\delta(z)= \begin{cases}+1, & z>0 \\ \pm 1, & z=0, \\ -1, & z<0\end{cases}
$$


$h(\cdot)$ is also a sign function defined as follows:

$$
h(z)= \begin{cases}+1, & z \geq 0 \\ -1, & z<0 .\end{cases}
$$

It can be seen from Equation (15) that both the cases $\xi_{T} \geq 0$ and $\xi_{T}<0$ are considered in the above closed form solution, which differs from [6]. Note that when $z=0$, either +1 or -1 can be chosen as the value of $\delta(0)$. The introduction of $\delta(\cdot)$ is to prevent the second term in the right hand side of Equation (15) from being eliminated. Moreover, the adding of the sign function $h(\cdot)$ can, to some extent, reduce the effect of time-to-go estimate on the guidance performance.

Combination of Equations (2), (13), and (15) yields

$$
\begin{aligned}
a_{m}= & N V \dot{\theta}+\left[\frac{3}{2} V \dot{\theta}+h\left(\xi_{T}\right) \delta(\sin \phi)\right. \\
& \cdot \sqrt{\left(\frac{3}{2} V \dot{\theta}\right)^{2}+\frac{3(N+1)(2 N-1) V^{5}}{R^{3}} \cdot\left|\xi_{T}\right|} .
\end{aligned}
$$

Equation (18) is the specific acceleration command of the proposed ITCG in this paper. It is shown in its expression that because the term $\left|\xi_{T}\right|$ instead of $\xi_{T}$ is used, the following inequality

$$
\left|\xi_{T}\right| \geq-\frac{3 \sin \phi^{2} R}{4(N+1)(2 N-1) V},
$$

always holds regardless of the sign of $\xi_{T}$. Therefore, Equation (14) always has real number solutions during the guidance process.

However, it should be noted that only the use of $\left|\xi_{T}\right|$ cannot guarantee the effectiveness of the proposed ITCG in the case of $\xi_{T}<0$. The sign function $h(\cdot)$ also plays an extremely important role in making the proposed ITCG differ from the ITCG of [6] in essence, which will be analyzed subsequently.

As already mentioned previously, the ITCG law becomes PNG when the additional acceleration $a_{\xi}$ is invalid, while a nonzero additional acceleration command generates a neighboring path perturbed with respect to the base trajectory generated by PNG. The combination of Equations (7) and (13) gives

$$
\eta=\eta_{0} \cdot\left(\frac{R}{R_{0}}\right)^{N-1}+a_{\xi} R_{0} \cdot g\left(\frac{R}{R_{0}}\right)
$$

where $g(\cdot)$ represents a shaping function of the trajectory, defined as follows:

$$
g\left(\frac{R}{R_{0}}\right)=\frac{R}{2 V^{2} R_{0}}\left[\left(\frac{R}{R_{0}}\right)^{N-2}-1\right] .
$$

Since the acceleration command $a_{\xi}$ can be considered as a proportional factor multiplied to $g(\cdot)$, as shown in Equa- tion (20), the perturbed shaping trajectory (i.e., the result of impact time control), to a large extent, relies on the value of $a_{\xi}$. To perform analysis, we remove the sign function $h(\cdot)$ from Equation (18) and replace $\left|\xi_{T}\right|$ by $\xi_{T}$, obtaining the same expression as that of [6]. And then by using Equations (2) and (6), the heading error rate can be written as follows:

$$
\begin{aligned}
\dot{\phi}= & -\frac{(N-1) V \sin \phi}{R}+\left[-\frac{3 V \sin \phi}{2 R}+\delta(\sin \phi)\right. \\
& \left.\cdot \sqrt{\left(-\frac{3 V \sin \phi}{2 R}\right)^{2}+\frac{3(N+1)(2 N-1) V^{3}}{R^{3}} \cdot \xi_{T}}\right] .
\end{aligned}
$$

For the case $\xi_{T}>0$, according to Equation (22) with the consideration of $\phi \in(0, \pi)$, it yields

$$
\dot{\phi}>-\frac{(N-1) V \sin \phi}{R}=(N-1) V \dot{\theta}
$$

On the other hand, considering $\phi \in(-\pi, 0)$, it gives

$$
\dot{\phi}<-\frac{(N-1) V \sin \phi}{R}=(N-1) V \dot{\theta} .
$$

We can see from Inequalities (23) and (24) that for the case $\xi_{T}>0$, the perturbed trajectory is longer than that of PNG. In contrast, in the case of $\xi_{T}<0$, the perturbed trajectory should be shorter than that of PNG. Obviously, the result of replacing $\xi_{T}$ (i.e., $\xi_{T}<0$ ) with $\left|\xi_{T}\right|$ is equivalent to the case of $\xi_{T}>0$, making Inequalities (23) and (24) still hold, which implies that the perturbed shaping trajectory that corresponds to the case $\xi_{T}<0$ cannot be achieved. Therefore, using the term $\left|\xi_{T}\right|$ instead of $\xi_{T}$ can only avoid the appearance of the complex command, which is the essential reason for the limitation of ITCG of [6] in the case of $\xi_{T}<0$.

On the contrary, by introducing the function $h(\cdot)$, the ITCG law proposed in this paper is effective for both cases $\xi_{T}>0$ and $\xi_{T}<0$. According to Equation (18), the heading error rate can be expressed as follows:

$$
\begin{aligned}
\dot{\phi}= & -\frac{(N-1) V \sin \phi}{R}+\left[-\frac{3 V \sin \phi}{2 R}+h\left(\xi_{T}\right) \delta(\sin \phi)\right. \\
& \cdot \sqrt{\left.\left(-\frac{3 V \sin \phi}{2 R}\right)^{2}+\frac{3(N+1)(2 N-1) V^{3}}{R^{3}} \cdot\left|\xi_{T}\right|\right]} .
\end{aligned}
$$

For the case $\xi_{T}<0$, considering $\phi \in(0, \pi)$, it yields

$$
\dot{\phi}<-\frac{(N-1) V \sin \phi}{R}-\frac{3 V \sin \phi}{R}=(N+2) V \dot{\theta} .
$$


For the case $\xi_{T}<0$, assuming $\phi \in(-\pi, 0)$, it gives

$$
\dot{\phi}>-\frac{(N-1) V \sin \phi}{R}-\frac{3 V \sin \phi}{R}=(N+2) V \dot{\theta} .
$$

It can be easily found from Inequalities (26) and (27) that the perturbed trajectory driven by Equation (18) is shorter than that induced by PNG, which corresponds to the requirement of the case $\xi_{T}<0$. For the case $\xi_{T}>0$, the performance of Equation (18) is as effective as that of [6].

In practical engineering, the seeker's target look angle can usually be considered as the heading error as long as the angle of attack of the missile is sufficiently small over the trajectory, which implies that the constraint on the look angle can be treated as the constraint on the heading error. Due to the limitation of the seeker's field-of-view, the heading error should satisfy the condition $\phi \in\left[-\phi_{\max }, \phi_{\max }\right]$ during the engagement (i.e., $\left.t \in\left[0, t_{f}\right]\right)$. In this paper, a logic that switches the additional acceleration commands when once the heading error exceeds the boundary is proposed. As a result, Equation (18) can be revised as follows:

$$
\begin{aligned}
a_{m}= & N V \dot{\theta}+s\left(\phi_{\max }-|\phi|\right)\left[\frac{3}{2} V \dot{\theta}+h\left(\xi_{T}\right) \delta(\sin \phi)\right. \\
& \cdot \sqrt{\left(\frac{3}{2} V \dot{\theta}\right)^{2}+\frac{3(N+1)(2 N-1) V^{5}}{R^{3}} \cdot\left|\xi_{T}\right|},
\end{aligned}
$$

where $s(\cdot)$ is the switching logic function which is chosen as follows:

$$
s(z)= \begin{cases}2\left(\frac{1}{1+e^{-\alpha z}}-\frac{1}{2}\right), & z \geq 0, \\ 0, & z<0,\end{cases}
$$

in which $\alpha$ is a positive constant (i.e., here we set $\alpha=20$ ). Note that this switching logic function is used to make the switching process smoother for practical applications. Due to the continuous variation of $\xi_{T}$ and $\sin \phi$ during the guidance process, it is unnecessary to smooth $h(\cdot)$ and $\delta(\cdot)$ using some certain logic functions.

\section{Theoretical Analysis}

In this section, theoretical analysis of the performance for the proposed ITCG will be preliminarily conducted, including the convergence of this guidance law, the dependence on time-to-go estimation, and the controllable range of the desired impact time, which is considered as the supplement to the preceding derivation.

4.1. Convergence. To explore the convergence of the proposed ITCG law with seeker's field-of-view constraint, we assume that the initial heading error satisfies $\phi_{0} \leq \phi_{\max }$ and then construct a candidate Lyapunov function as follows:

$$
V_{L}(\phi)=\frac{1}{2} \phi^{2}
$$

Differentiating Equation (30) with respect to time $t$, it yields

$$
\dot{V}_{L}(\phi)=\phi \cdot \dot{\phi}=\phi\left(\frac{a_{m}}{V}+\frac{V \sin \phi}{R}\right) .
$$

Note that from physical perspective, the value of $R$ is certainly positive during the guidance process, thus the dynamics on $R$ has no effect on the convergence analysis.

Substitution of Equations (2) and (28) into Equation (31) gives

$$
\begin{aligned}
\dot{V}_{L}(\phi)= & \frac{-(N-1) V \phi \sin \phi}{R}+\phi s\left(\phi_{\max }-|\phi|\right) \\
& \cdot\left[-\frac{3 V \sin \phi}{2 R}+h\left(\xi_{T}\right) \delta(\sin \phi)\right. \\
& \left.\cdot \sqrt{\left(-\frac{3 V \sin \phi}{2 R}\right)^{2}+\left(\frac{V}{R}\right)^{3} 3(N+1)(2 N-1)\left|\xi_{T}\right|}\right]
\end{aligned}
$$

Evidently, for the case $\xi_{T}<0, \dot{V}_{L}(\phi)<0$ always holds because $\phi \cdot \delta(\sin \phi) \geq 0$ and $h\left(\xi_{T}\right)<0$. For the special case $\xi_{T}=0$, the proposed ITCG law evolves into PNG, making $\dot{V}_{L}(\phi)<0$ hold all the time. Thus, for the case $\xi_{T} \leq 0, \dot{V}_{L}(\phi)$ is always negative definite and, as a result, the value of $|\phi|$ decreases monotonically during the guidance process. According to the condition $\phi_{0} \leq \phi_{\max }$, it is obvious that the proposed ITCG law is able to deal with the filed-of-view constraint.

For the case $\xi_{T}>0$, the situation $\dot{V}_{L}(\phi)>0$ is likely to occur at initial stage, making the value of $|\phi|$ increases. As already mentioned, $\dot{V}_{L}(\phi)$ is presented as a function of the heading error $\phi$. When we assume $|\phi|=\phi_{\max }$, the following expression

$$
\left.\dot{V}_{L}(\phi)\right|_{|\phi|=\phi_{\max }}=\frac{-(N-1) V \phi_{\max } \sin \phi_{\max }}{R}<0,
$$

can be obtained, which indicates that $\dot{V}_{L}(\phi)$ is negative definite near the boundary of the seeker's field-of-view. Note that because $\dot{V}_{L}(\phi)$ is continuous with respect to $\phi$ and $\dot{V}_{L}\left(\phi_{\max }\right)<0$, there exists a certain positive constant $\mu$ that can make $\dot{V}_{L}\left(\phi_{\max }-\mu\right)=0$ hold. Under this circumstance, the acceleration command turns to $a_{m}=V \dot{\theta}$, which implies that the value of $|\phi|$ cannot exceed $\phi_{\max }$ over the engagement (i.e., $t \in\left[0, t_{f}\right]$ ) provided that the initial heading error is smaller than the boundary value (i.e., $\left.\phi_{0} \leq \phi_{\max }\right)$. If and only if $\phi_{0}=\phi_{\max }$, the value of $|\phi|$ can reach the level of $\phi_{\max }$. Therefore, the presented ITCG law is capable of controlling the impact time of the missile under the field-of-view constraint. 
Furthermore, it can be found from Equations (28) and (29) that even if the initial heading error is greater than the boundary value (i.e., $\left.\phi_{0}>\phi_{\max }\right), \dot{V}_{L}(\phi)<0$ still holds and the proposed ITCG law turns into PNG, which implies that the heading error of the missile will decrease to the boundary within a certain period prior to implementing the impact time control. In this sense, it allows the presented ITCG law to adapt itself to some extreme conditions.

4.2. Dependence on Time-to-Go Estimation. As mentioned in the preceding sections, the additional acceleration command $a_{\xi}$ is expressed as a function of the impact time error that depends on the time-to-go estimation. For the proportionalnavigation-based ITCG law, an inaccurate time-to-go leads to an inaccurate neighboring path perturbed with respect to the base trajectory generated by PNG. Even if the impact time error is greater than zero (i.e., $\xi_{T}>0$ ) at the beginning of the flight, the practical trajectory will deviate and never come back to the desired trajectory due to a time-to-go estimation without sufficient accuracy. Meanwhile, the impact time error tends to less than zero, making the acceleration command becomes a complex. In this circumstance, the ITCG of [6] cannot work over the engagement, which indicates that the acceleration command is significantly affected by the time-to-go estimation error. To solve this problem, more accurate higher-order terms of time-to-go estimate of PNG were used in [6]. For the proposed ITCG law, the missile is able to come back to the required trajectory from the deviated one by switching the commands between $\xi_{T} \geq 0$ and $\xi_{T}<0$, resulting in lower dependence on the time-to-go estimation in contrast to that presented in [6], as will be demonstrated in Section 5. However, the strategy of switching guidance commands may probably result in an unexpected tremble. To overcome this deficiency, the guidance law Equation (28) is improved by introducing a smooth function, i.e.,

$$
\begin{aligned}
a_{m}= & N V \dot{\theta}+w\left(\xi_{T}\right) s\left(\phi_{\max }-|\phi|\right)\left[\frac{3}{2} V \dot{\theta}+h\left(\xi_{T}\right) \delta(\sin \phi)\right. \\
& \left.\cdot \sqrt{\left(\frac{3}{2} V \dot{\theta}\right)^{2}+\frac{3(N+1)(2 N-1) V^{5}}{R^{3}} \cdot\left|\xi_{T}\right|}\right]
\end{aligned}
$$

where the smooth function $w(\cdot)$ is defined as follows:

$$
w(z)= \begin{cases}0, & |z|<k_{1} \\ \frac{|z|-k_{1}}{k_{2}-k_{1}}, & k_{1} \leq|z| \leq k_{2} \\ 1, & |z|>k_{2},\end{cases}
$$

where $k_{1}$ and $k_{2}$ are small positive constants and $k_{2}>k_{1}$. Note that large values of $k_{1}$ and $k_{2}$ should be considered when the time-to-go estimation is not sufficiently accurate. On the other hand, the smooth function is unnecessary when the accuracy of time-to-go estimation is sufficient.
Note that through substituting Equations (2) and (34) into Equation (31), it can be found that the introduction of $w(\cdot)$ has no impact on the convergence that has already been proved in Section 4.1. For the sake of brevity, the detailed derivation is omitted here.

4.3. Controllable Range of the Desired Impact Time. Although the ITCG law proposed in this paper is effective for both cases $\xi_{T}>0$ and $\xi_{T}<0$, the desired impact time cannot be set arbitrarily. To better understand the characteristics of this guidance law, it is necessary to analyze the controllable range of the desired impact time.

Assuming that the time-to-go estimation is sufficiently accurate, we can see from Equations (23) and (24) that the minimum available impact time $t_{f \min }$ satisfies the following inequality

$$
t_{f \min }<t_{N}
$$

where $t_{N}$ is the time of flight driven by PNG with a navigation gain of $(N+3)$.

Obviously, the minimum impact time that is achievable in practice must be greater than the time as the missile flies along the straight line between the missile and the target (i.e., denoted as $t_{M}=R_{0} / V$ ). Thus, the range of the minimum impact time $t_{f \min }$ can be described as follows:

$$
t_{f \min } \in\left(t_{M}, t_{N}\right)
$$

According to Equation (1), when $\phi>90^{\circ}$ or $\phi=\phi_{\max }$, $\dot{R} \geq 0$ always holds. Theoretically, if we do not consider the seeker's filed-of-view constraint or the boundary value is greater than 90 degrees, the maximum available impact time $t_{f \text { max }}$ has no upper bound (i.e., infinity). On the contrary, the maximum impact time $t_{f \text { max }}$ that is achievable is bounded when the boundary of the field-of-view angle is less than 90 degrees. Sufficient detail of an approach that determines $t_{f \text { max }}$ for the case $\phi_{\max }<90^{\circ}$ is presented in [7].

\section{Numerical Simulation and Results}

In this section, the proportional-navigation-based ITCG law presented in Section 3 is validated by numerical simulations and its performance is also compared to those obtained in earlier studies $[6,8]$. To this end, the higher-order time-togo estimate of PNG given by [6] is restated as follows:

$$
\begin{aligned}
t_{\mathrm{go} P N}= & \frac{R}{V}\left[1+\frac{\sin ^{2} \phi}{2(2 N-1)}+\frac{3 \sin ^{4} \phi}{8(4 N-3)}\right. \\
& \left.+\frac{5 \sin ^{6} \phi}{16(6 N-5)}+\frac{35 \sin ^{8} \phi}{128(8 N-7)}+\cdots\right] .
\end{aligned}
$$

In the rest of this section, various forms of Equation (38) in terms of the order of $\sin \phi$ are used. For instance, the $n$ thorder approximation ( $n$ is an integer) refers to the expression whose highest-order term is $\sin ^{n} \phi$. Note that the zeroth- 


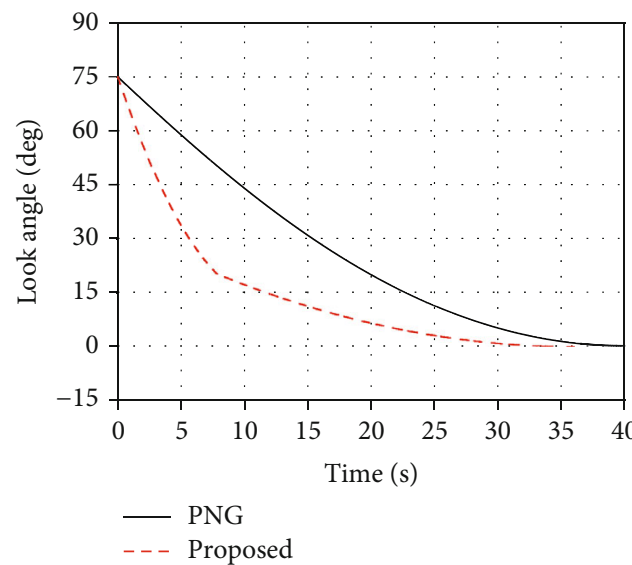

(a) Look angle vs. time

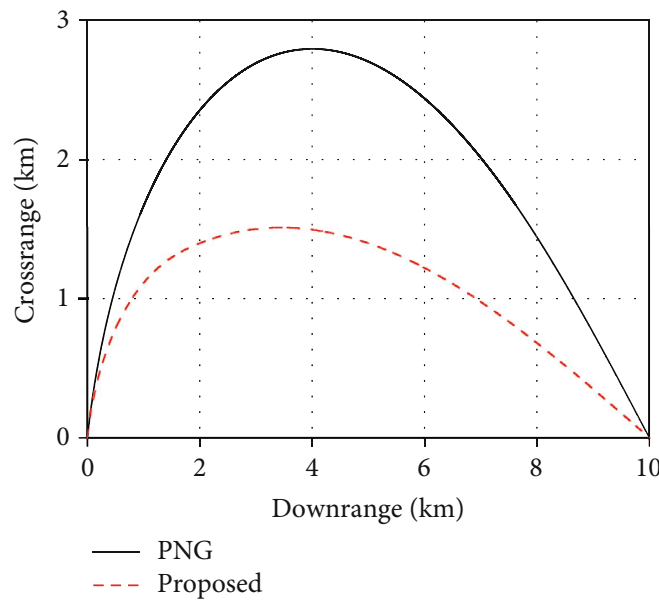

(c) Variation of trajectory

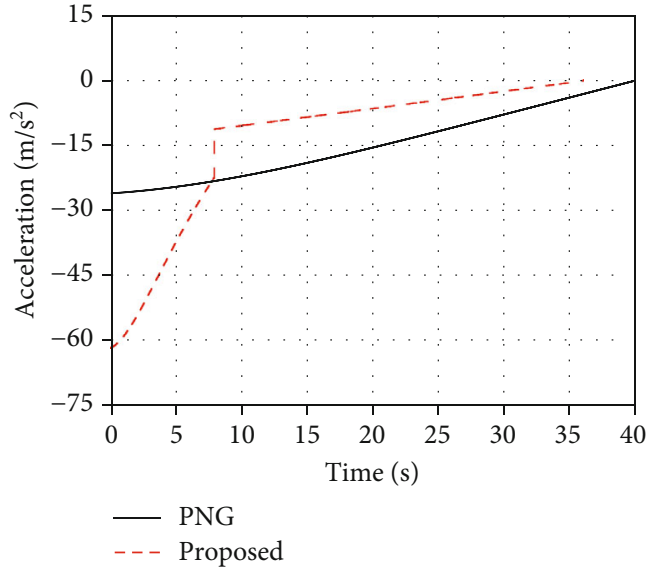

(b) Acceleration command vs. time

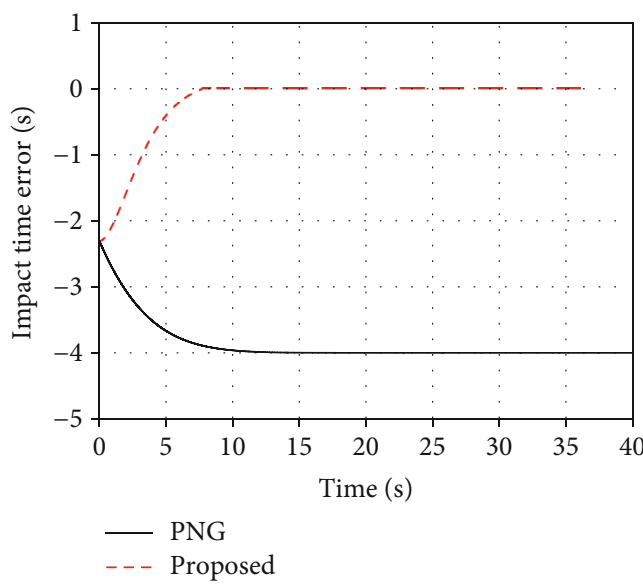

(d) Impact time error vs. time

FIGURE 2: Simulation results for the case $\xi_{T}<0\left(t_{f}=36 \mathrm{~s}\right)$.

order approximation is the simplest formula that estimates the time-to-go using the range divided by the closing speed.

5.1. A Specific Case for Validation. As already mentioned, because the presented ITCG is as effective as that of [6] in the case of $\xi_{T}>0$, similar simulations are not repeated here. Instead, we focus on the case $\xi_{T}<0$ and consider the following conditions: $N=3, R_{0}=10000 \mathrm{~m}, V=300 \mathrm{~m} / \mathrm{s}, \phi_{0}=75$ deg, $\theta_{0}=0 \mathrm{deg}$, and the impact time of the PNG trajectory is $40 \mathrm{~s}$. Moreover, we set the desired impact time as $t_{f}=36 \mathrm{~s}$, choose $k_{1}=0.0005$ and $k_{2}=0.001$, and apply the sixthorder time-to-go approximation of Equation (38) to the ITCG law Equation (34). The simulation results compared with that of PNG are shown in Figure 2.

As shown in Figure 2, the desired impact time can be accurately achieved by using the proposed ITCG law. It is illustrated in Figure 2(b) that the initial acceleration command provided by Equation (34) is greater than that of PNG, leading to a heading error rate faster than that of PNG. As a result, a neighboring path shorter than the trajectory of PNG is generated, as shown in Figure 2(c), and then the impact time error successfully converges to zero (i.e., the desired impact time is achieved). This result corresponds to the preceding analysis. In addition, we can see from Figure 2(d) that although the impact time error driven by PNG cannot converge to zero, it is capable of leveling off at a constant value (i.e., approximately $4 \mathrm{~s}$ ). This phenomenon can be primarily attributed to the fact that the accuracy of the sixth-order time-to-go approximation of Equation (38) gradually increases as the look angle decreases.

5.2. Effect of Time-to-Go Estimation on the Proposed ITCG. This subsection attempts to examine the effect of time-togo estimation on the proposed ITCG law through numerical simulations. To this purpose, we introduce the accurate timeto-go estimation formula in the framework of PNG expressed by the incomplete beta function. This accurate time-to-go formula, as presented in [5], is called the exact solution to the time-to-go of PNG.

We consider the following simulation conditions: $N=3$, $R_{0}=10000 \mathrm{~m}, V=300 \mathrm{~m} / \mathrm{s}, \phi_{0}=45 \mathrm{deg}, \theta_{0}=0 \mathrm{deg}$, and the impact time of the PNG trajectory is $35.50 \mathrm{~s}$. In the following simulation, we choose the desired impact time as $t_{f}=50 \mathrm{~s}$. The results obtained through applying the exact solution, the fourth- and sixth-order approximations of Equation (38) to the proposed ITCG law are shown in Figures 3 and 


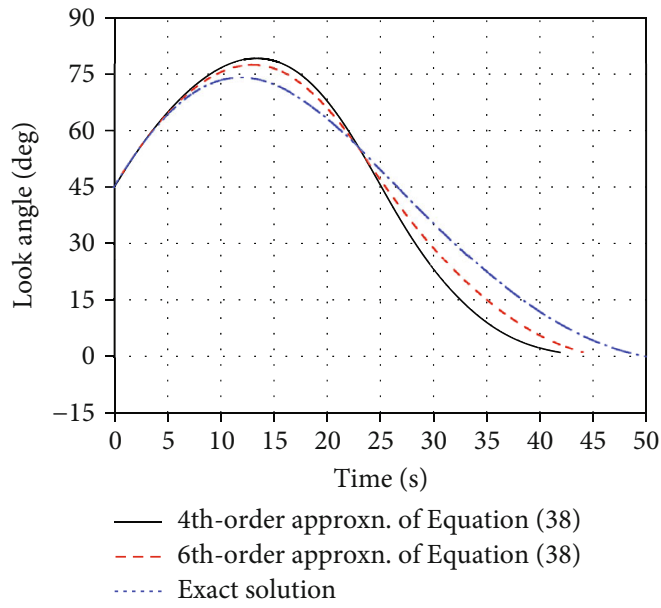

(a) Look angle vs. time

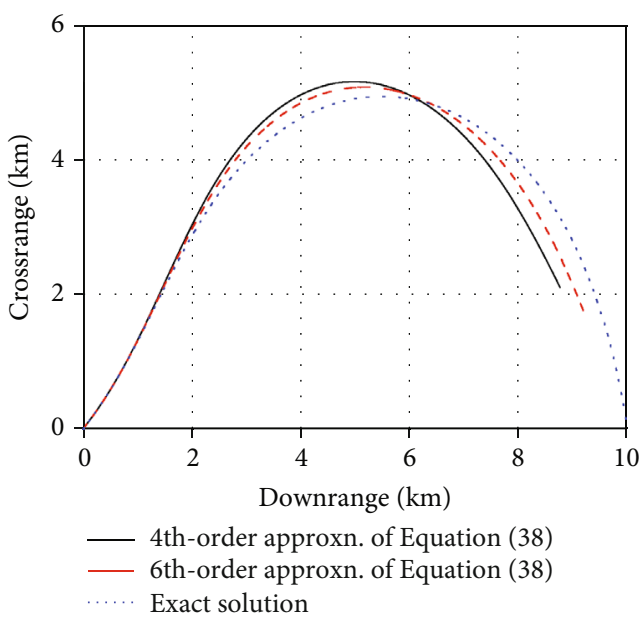

(c) Variation of trajectory

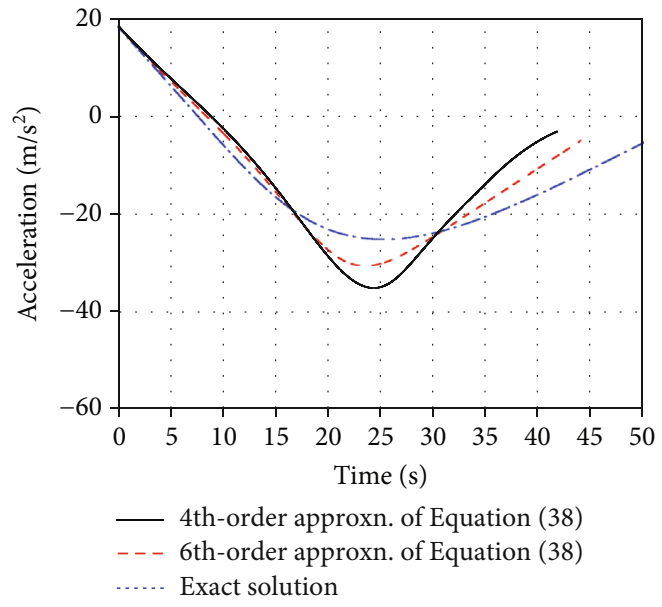

(b) Acceleration command vs. time

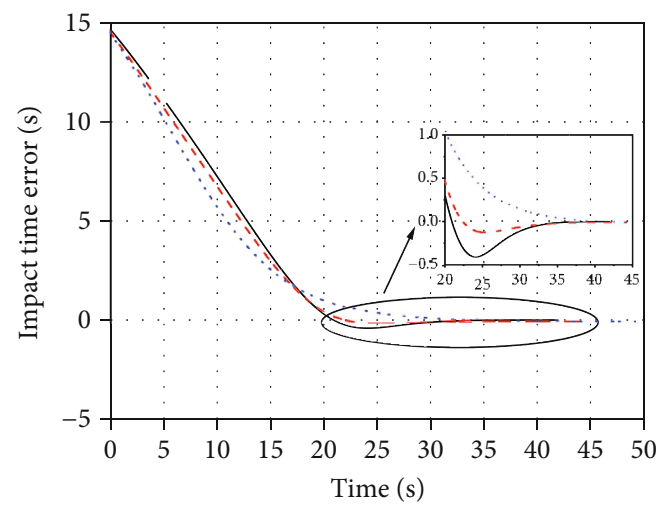

- 4th-order approxn. of Equation (38)
-- 6th-order approxn. of Equation (38)
$\ldots$... Exact solution

(d) Impact time error vs. time

FIgURE 3: Results of ITCG law of [6] using different time-to-go estimation formulas.

4. Note that because the exact solution is sufficiently accurate, Equation (28) instead of (34) is used for numerical simulations when the exact solution is adopted. When the fourthand sixth-order approximations of Equation (38) are adopted, Equation (34) is used to perform simulations (e.g., we choose $k_{1}=0.0005$ and $\left.k_{2}=0.001\right)$.

As shown in Figures 3 and 4, when the exact solution is applied, both the ITCG laws proposed in [6] and in this effort perform well. However, when the fourth- and sixth-order approximations are adopted, the ITCG law of [6] fails to achieve the desired impact time whereas the proposed ITCG law succeeds in controlling the impact time. Due to the deficient accuracy of Equation (38) for estimating time-to-go, the acceleration command induced by the ITCG of [6] becomes a complex at some certain moment during the missile's flight. it is found from these figures that more accurate time-to-go estimate results in gently varying acceleration command, relatively smaller maximum look angle during the guidance process, and a closer trajectory corresponding to exact impact time control. For the ITCG of [6], inaccurate timeto-go estimation makes the flight path deviates from the exact trajectory. On the contrary, by using the function $h\left(\xi_{T}\right)$ with respect to the impact time error that appears in Equation (34), the deviated flight path can be corrected and ultimately return to the exact trajectory. Therefore, it is demonstrated that the dependence on the time-to-go estimation of the proposed ITCG is much lower than that of the ITCG in [6], which is in accordance with the previous analysis.

To make a further examine, the controllable ranges of the desired impact time are calculated by a trial-and-error approach for the aforementioned simulation condition using various approximations of Equation (38) and the exact solution. Note that for convenience, the impact time is considered to be controllable when the distance between the missile and the target is less than $0.2 \mathrm{~m}$, and meanwhile, the magnitude of the impact time error is less than a specified value. The results of the controllable ranges of the desired impact time are listed in Table 1.

We can see from Table 1 that the ITCG of [6] does not work when the zeroth-order approximation of Equation (38) is used. By using the ITCG of [6], the minimum controllable impact time is equal to the impact time of the PNG trajectory (i.e., $35.50 \mathrm{~s}$ ) and the maximum controllable impact time grows somewhat as the accuracy of the time-to-go 

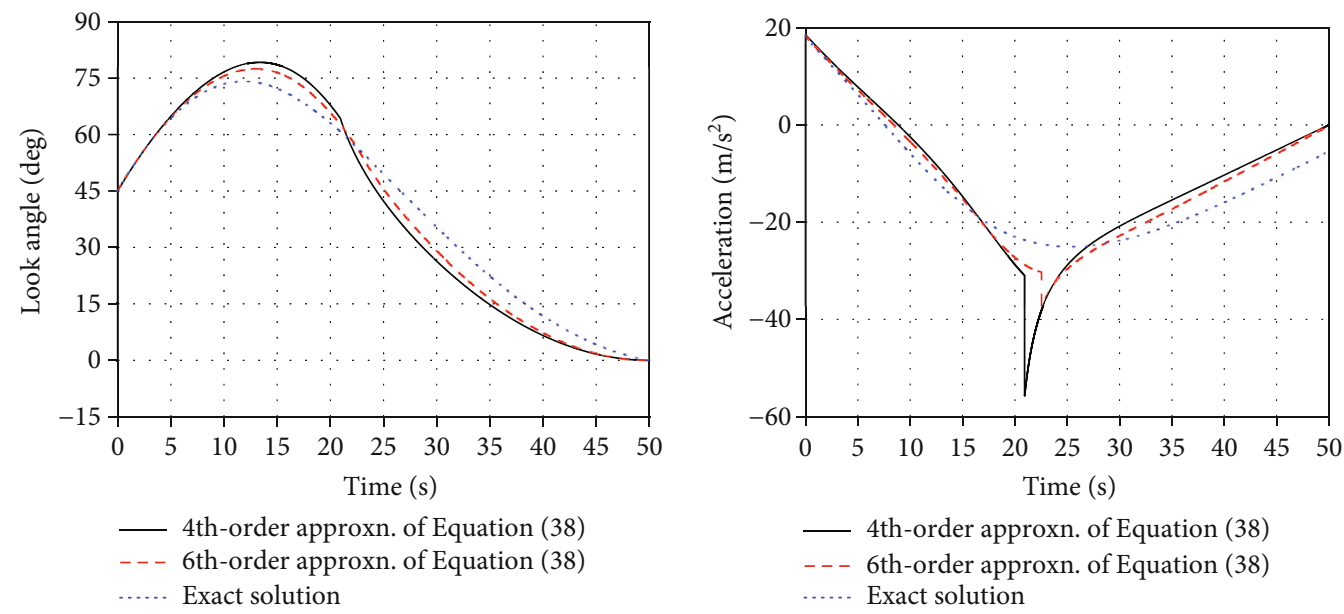

_ 4th-order approxn. of Equation (38)

….. Exact solution

(a) Look angle vs. time

(b) Acceleration command vs. time
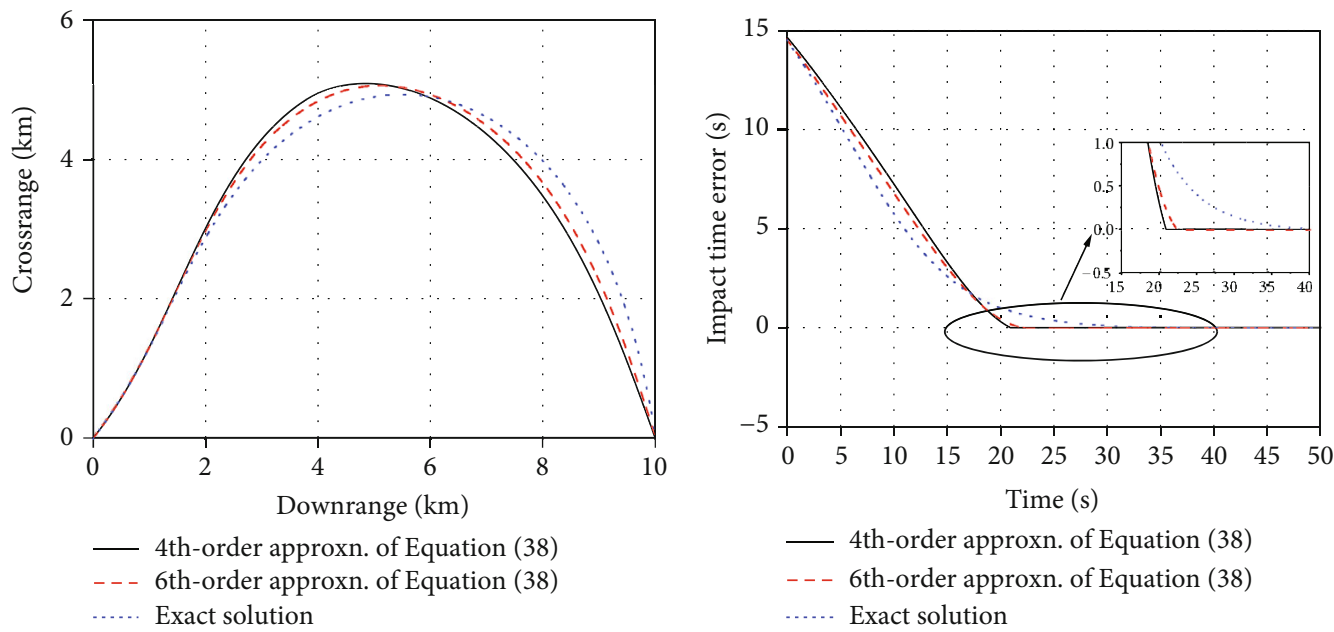

(d) Impact time error vs. time

(c) Variation of trajectory

FIgURE 4: Results of proposed ITCG law using different time-to-go estimation formulas.

TABLE 1: Comparison of controllable ranges of the desired impact time.

\begin{tabular}{lccc}
\hline Time-to-go estimation formula & ITCG law in [6] & Proposed ITCG law & Impact time error $\xi_{T}(\mathrm{~s})$ \\
\hline Zeroth-order approximation of Equation (38) & $\mathrm{N} / \mathrm{A}^{\mathrm{a}}$ & $35.09 \mathrm{~s} \sim 38.80 \mathrm{~s}$ & $<0.4$ \\
Second-order approximation of Equation (38) & $35.50 \mathrm{~s} \sim 40.58 \mathrm{~s}$ & $34.17 \mathrm{~s} \sim 52.48 \mathrm{~s}$ & $<10^{-3}$ \\
Fourth-order approximation of Equation (38) & $35.50 \mathrm{~s} \sim 6.17 \mathrm{~s}$ & $34.15 \mathrm{~s} \sim 56.69 \mathrm{~s}$ & $<10^{-3}$ \\
Sixth-order approximation of Equation (38) & $35.50 \mathrm{~s} \sim 48.90 \mathrm{~s}$ & $34.15 \mathrm{~s} \sim 58.30 \mathrm{~s}$ & $<10^{-3}$ \\
Exact solution & $35.50 \mathrm{~s} \sim$ infinity & $34.15 \mathrm{~s} \sim$ infinity & $<10^{-3}$ \\
\hline
\end{tabular}

${ }^{a}$ N/A stands for "not applicable."

estimate increases. For the second-, fourth-, and sixth-order approximations of Equation (38), the interval lengths of the desired impact time obtained by the ITCG of [6] are $5.08 \mathrm{~s}$, $10.67 \mathrm{~s}$, and $13.40 \mathrm{~s}$, respectively. By using the proposed ITCG, the desired impact time smaller than that of the PNG trajectory can also be achieved. Correspondingly, the interval lengths are $18.31 \mathrm{~s}, 22.54 \mathrm{~s}$, and $24.15 \mathrm{~s}$ for the second-, fourth-, and sixth-order approximations, respectively, which demonstrates that the controllable range of the proposed ITCG law is fairly wider than that of [6]. As already analyzed, with the help of the exact solution, the upper bounds of the available impact time for these two laws can be considered as infinity. However, even if the exact solution is used, the ITCG of [6] still fails to achieve the impact time smaller than that of the PNG trajectory. According to the above simulation conditions, the lower and upper bounds of $t_{f \min }$ are $t_{M}=33.33 \mathrm{~s}$ and $t_{N}=34.30 \mathrm{~s}$, respectively. Hence, all the lower bound values of the impact time achieved by the proposed ITCG law satisfy Equation (36) except for the case where the zeroth-order approximation is used. 


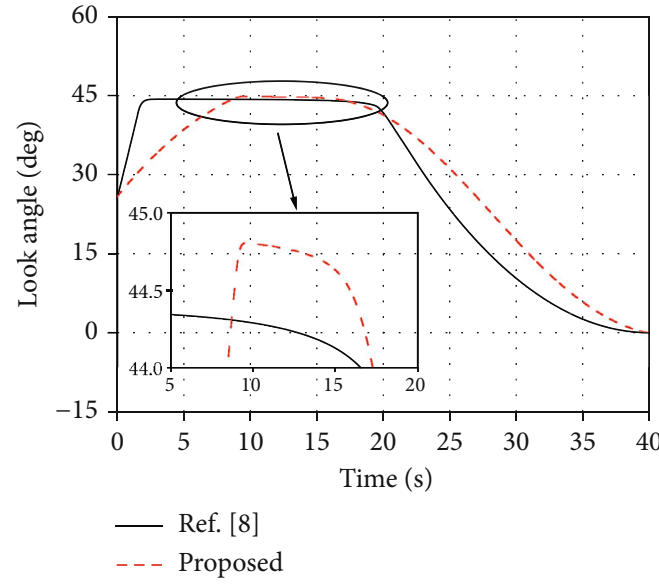

(a) Look angle vs. time

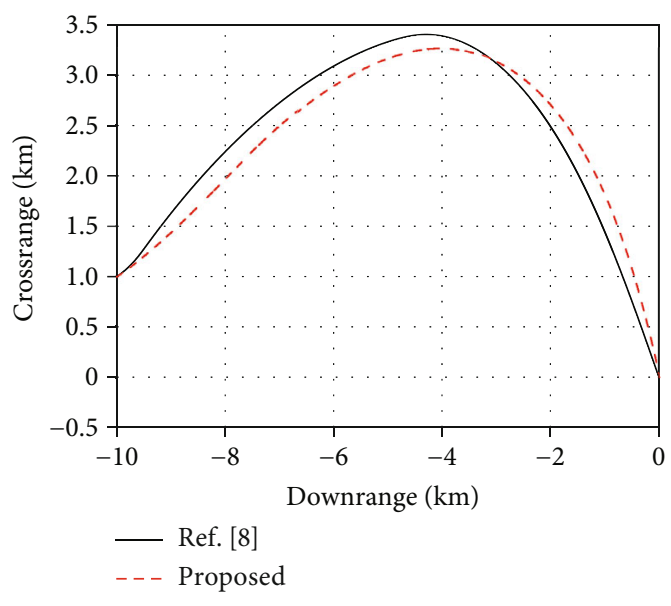

(c) Variation of trajectory

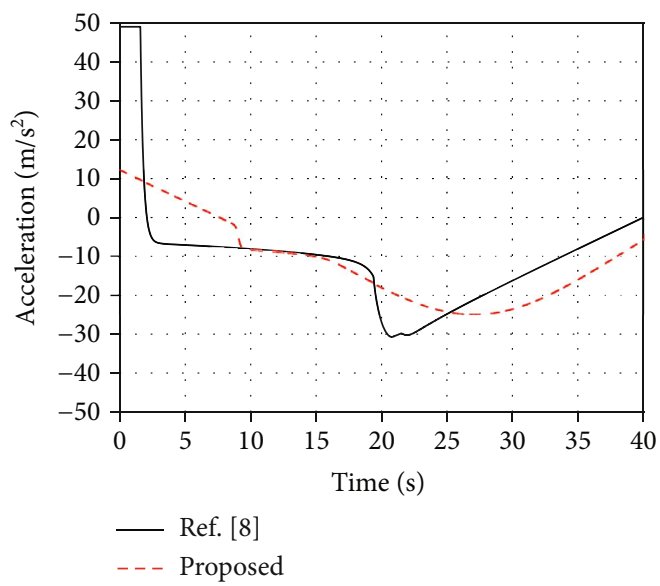

(b) Acceleration command vs. time

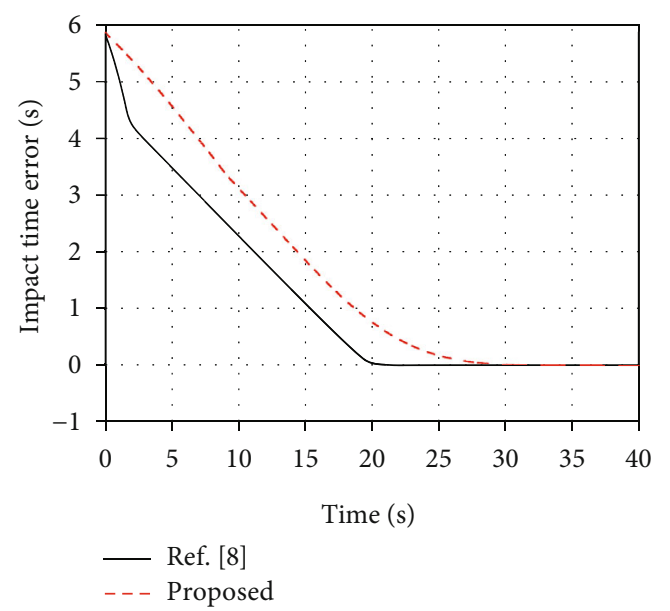

(d) Impact time error vs. time

Figure 5: Simulation of ITCG laws with field-of-view constraints.

It is also shown in Table 1 that the impact time errors obtained by using these two laws are less than $0.001 \mathrm{~s}$. Applying the proposed ITCG law to the issues with regard to salvo attack and/or cooperative attack, the flexibility may probably be augmented. It is worth noting that the proposed ITCG law can even be applicable to the case using the zeroth-order approximation whereas the ITCG of [6] is not applicable. In this special case, the desired impact time can also be set larger or smaller than the impact time of the PNG trajectory, although the controllable range is relatively narrow and the impact time error is slightly large (i.e., less than $0.4 \mathrm{~s}$ ). In this sense, the proposed ITCG law is able to eliminate the singularity driven by the inaccuracy of the time-to-go estimate.

5.3. Several Cases with Field-of-View Constraint. To validate the feasibility of the application of the proposed ITCG to the cases with field-of-view constraint, we consider the following condition: $N=3$, the missile's position $\left(x_{M}, y_{M}\right)=$ $(-10000,1000)$, the target's position $\left(x_{T}, y_{T}\right)=(0,0), V=$ $300 \mathrm{~m} / \mathrm{s}, \quad \phi_{0}=25.7 \mathrm{deg}, \quad \phi_{\max }=45 \mathrm{deg}$, and the desired impact time $t_{f}=40 \mathrm{~s}$.
Note that because the acceleration of a missile cannot be infinite in practice, we set such a saturated acceleration $a_{\max }=48 \mathrm{~m} / \mathrm{s}^{2}$ that any practical acceleration greater than $a_{\max }$ is limited to the value of $a_{\max }$. The simulation results are shown in Figure 5, where the results obtained using this proposed ITCG law are compared to those given by [8].

We can see from Figure 5 that the proposed ITCG law at least has comparable results to that presented in [8]. The maximum heading error driven by the proposed ITCG law appears to be a little greater (i.e., approximately $0.5 \mathrm{~s}$ ) than that induced by [8]. However, the duration of the look angle staying at the field-of-view boundary in [8] is longer, as shown in Figure 5(a). The initial acceleration command and the maximum acceleration over the engagement driven by the proposed guidance law are quite smaller in comparison with that in [8], as illustrated in Figure 5(b). At the beginning, the acceleration driven by [8] is saturated (i.e., exceeds $48 \mathrm{~m} / \mathrm{s}^{2}$ ) whereas the acceleration of the proposed guidance law is approximately $12 \mathrm{~m} / \mathrm{s}^{2}$. Generally speaking, the variations of the trajectory and the impact time error 


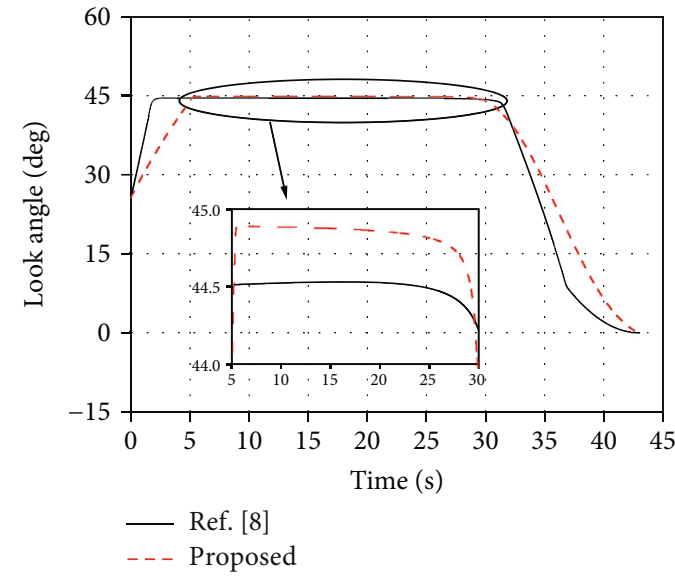

(a) Look angle vs. time

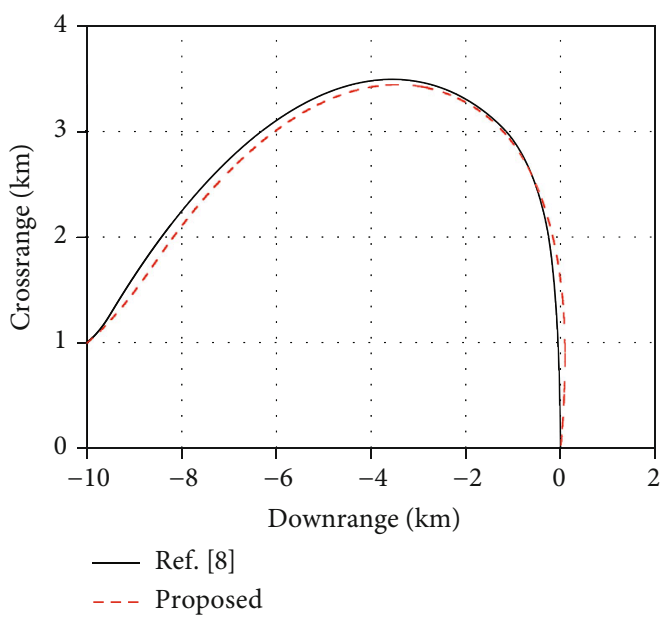

(c) Variation of trajectory

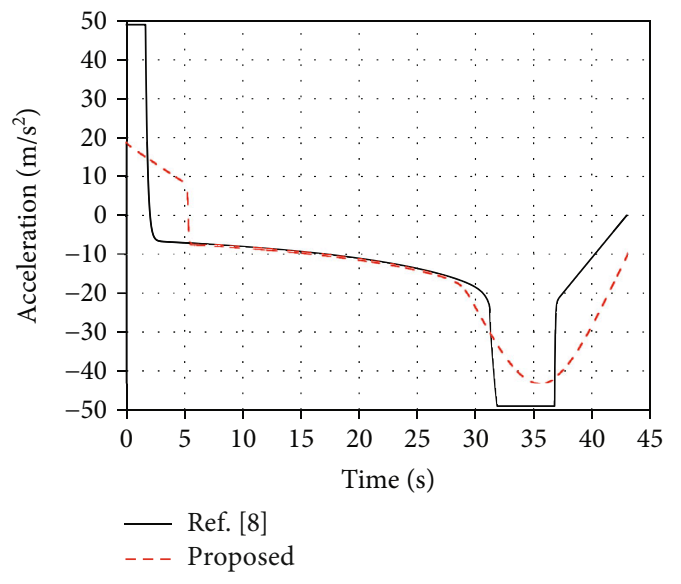

(b) Acceleration command vs. time

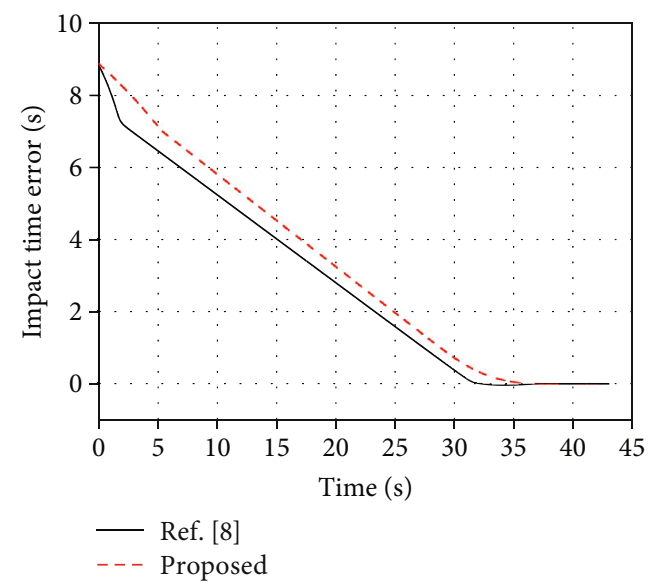

(d) Impact time error vs. time

FIgURE 6: Simulation of ITCG laws in the case of $t_{f}=43 \mathrm{~s}$ with field-of-view constraint.

provided by these two laws are similar, as illustrated in Figures 5(c) and 5(d).

To make a deep comparison with the ITCG of [8], here, we consider another case where the desired impact time $t_{f}=43 \mathrm{~s}$. As mentioned in [8], the impact time of $t_{f}=43 \mathrm{~s}$ appears to be the boundary value of the controllable range of the desired impact time. The results obtained using these two ITCG laws are shown in Figure 6.

As illustrated in Figure 6, the main difference between the proposed ITCG law and the ITCG of [8] comes from the acceleration command. Similar to Figure 6(b), the initial acceleration command driven by the proposed ITCG law is much smaller. In addition, by using the ITCG of [8], the saturation phenomenon also occurs within a certain period (e.g., ranging from $t=32 \mathrm{~s}$ to $t=36 \mathrm{~s}$ ), whereas the acceleration of the proposed ITCG in the same period does not exceed $45 \mathrm{~m} / \mathrm{s}^{2}$.

As mentioned previously, the ITCG law presented in $[8,9]$ does not work when the initial heading error is equal to zero. To validate that the proposed ITCG is capable of eliminating this singularity, we perform another simulation with $\phi_{0}=0$ deg and $t_{f}=39 \mathrm{~s}$ and compare the results with those obtained in [8]. The simulation results are shown in Figure 7.

We can see from Figure 7 that because the ITCG of [8] is not adapted to the case with zero initial heading error, the acceleration command as well as the look angle is zero from the beginning to the end of the engagement and, obviously, the missile flies along the straight line between the missile and the target. Meanwhile, the proposed ITCG law successfully eliminates the aforementioned singularities and, as a consequence, achieves the desired impact time.

\section{Conclusions}

This paper presents a nonsingular proportional-navigationbased impact time control guidance law with the consideration of the seeker's field-of-view constraint based on nonlinear formulations. Using numerical simulations, it is demonstrated that the proposed ITCG law is capable of achieving the desired impact time larger or smaller than the impact time of the PNG trajectory and alleviating its dependence on the time-to-go estimation by switching the commands according to the sign of the impact time error. In 


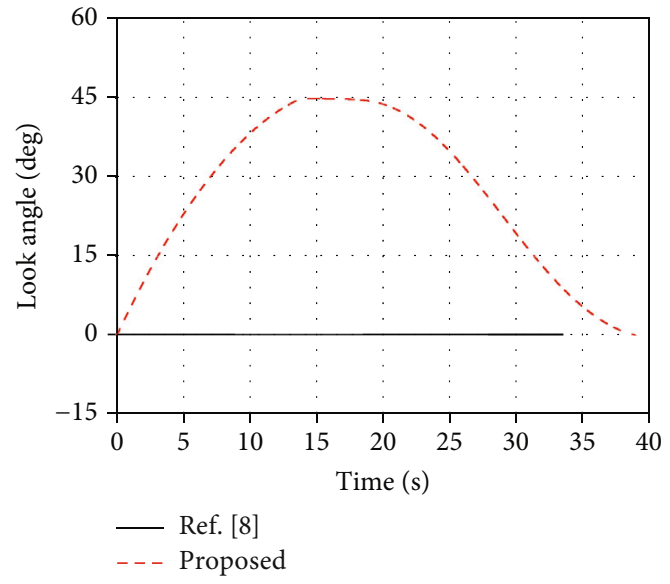

(a) Look angle vs. time

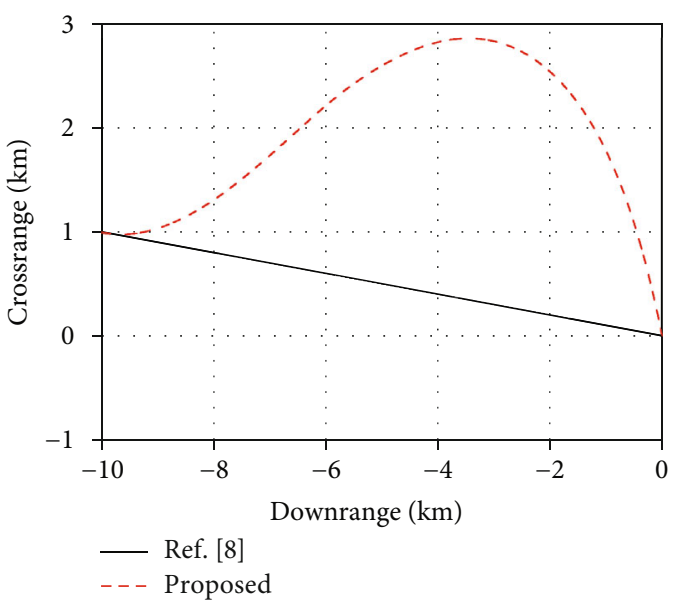

(c) Variation of trajectory

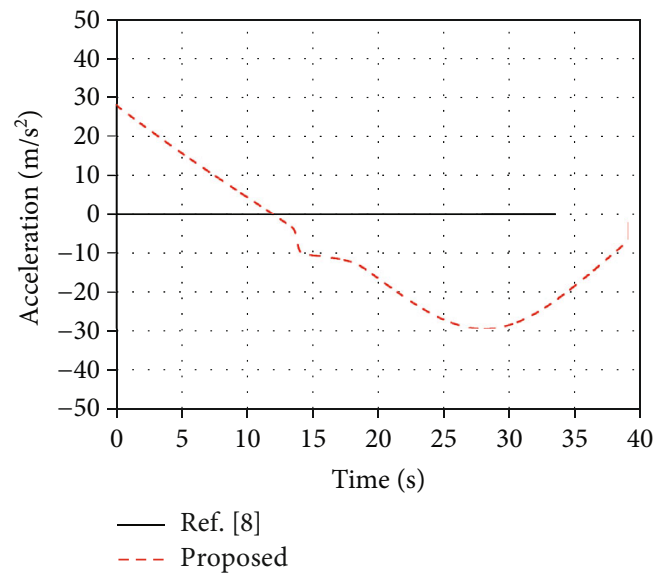

(b) Acceleration command vs. time

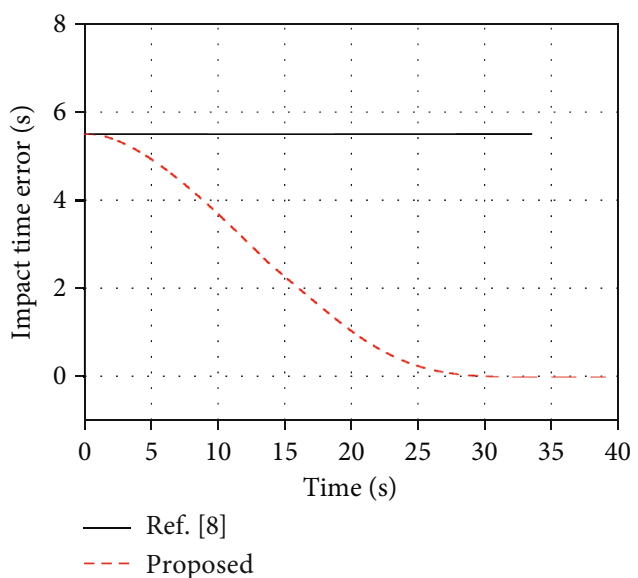

(d) Impact time error vs. time

Figure 7: Simulation of ITCG laws with field-of-view constraint ( $\left.\phi_{0}=0 \mathrm{deg} ; t_{f}=39 \mathrm{~s}\right)$.

some cases, the proposed ITCG law is shown to be superior over some other guidance laws presented in earlier studies in that it guarantees the guided trajectory from divergence over the engagement, showing its augmented robustness and flexibility. To address the field-of-view constraint, a switching logic pertinent to the additional acceleration command is incorporated into the total acceleration command. It is also validated by numerical simulations that the ITCG law is applicable to the constraints associated with the saturated acceleration command, the initial heading error, and the seeker's field-of-view angle. In summary, the PNG-based ITCG law with lower dependence on time-to-go estimation extends the controllable range of the desired impact time and adapts itself to more conditions in comparison with some other PNG-based ITCG laws in earlier studies. From practical point of view, this proposed law aids in taking full advantage of the characteristics of PNG and is expected to be supplementary to the current research literature.

\section{Notations}

$a_{b}: \quad$ Feedback acceleration command

$a_{m}$ : Total acceleration command $a_{\xi}: \quad$ Additional acceleration command

$g(\cdot)$ : A shaping control function, defined as Equation (22)

$H$ : Hamilton function

$h(\cdot)$ : C sign function, defined as Equation (18)

$J$ : control effort

$k_{1}, k_{2}$ : Positive constants used to determine the smooth function $w(\cdot)$

$N: \quad$ Navigation gain

$R: \quad$ Distance between the missile and the target

$R_{0}$ : Initial distance between the missile and the target

$s(\cdot)$ : A switching logic function, defined as Equation (30)

$t$ : Time

$t_{f}: \quad$ Impact time

$t_{f d}: \quad$ Real time-to-go

$t_{f \text { min }}:$ Minimum impact time

$t_{f \text { max }}:$ Maximum impact time

$t_{\mathrm{goPN}}:$ Predicted time-to-go

$t_{M}: \quad$ Time of flight, defined as $t_{M}=R_{0} / V$

$t_{N}: \quad$ Time of flight driven by PNG

$V: \quad$ Speed of the missile

$V_{L}(\cdot)$ : Lyapunov function

$w(\cdot)$ : Smooth function, defined as Equation (36) 
$x, y$ : Downrange and crossrange of trajectory

$\alpha: \quad$ A positive constant, shown in Equation (30)

$\gamma: \quad$ Flight path angle

$\delta(\cdot): \quad$ A sign function, defined as Equation (17)

$\theta: \quad$ Line-of-sight angle

$\theta_{0}: \quad$ Initial line-of-sight angle

$\lambda_{\eta}: \quad$ Lagrange multiplier

$\xi_{T}: \quad$ Impact time error

$\phi$ : $\quad$ Heading error

$\phi_{0}$ : Initial heading error

$\phi_{\max }$ : Boundary of the field-of-view angle.

\section{Data Availability}

The data used to support the findings of this study are available from the corresponding author upon request.

\section{Conflicts of Interest}

The authors declare that they have no conflicts of interest.

\section{Acknowledgments}

This work is supported by the National Natural Science Foundation of China (number 11402117).

\section{References}

[1] I.-S. Jeon, J.-I. Lee, and M.-J. Tahk, "Impact-time-control guidance law for anti-ship missiles," IEEE Transactions on Control Systems Technology, vol. 14, no. 2, pp. 260-266, 2006.

[2] I. S. Jeon, J. I. Lee, and M. J. Tahk, "Homing guidance law for cooperative attack of multiple missiles," Journal of Guidance, Control, and Dynamics, vol. 33, no. 1, pp. 275-280, 2010.

[3] S. Ghosh, D. Ghose, and S. Raha, "Three dimensional PN based impact angle control for higher speed nonmaneuvering targets," in 2013 American Control Conference, Washington, DC, USA, 2013.

[4] Y. Zhang, X. Wang, and G. Ma, "Impact time control guidance law with large impact angle constraint," Proceedings of the Institution of Mechanical Engineers, Part G: Journal of Aerospace Engineering, vol. 229, no. 11, pp. 2119-2131, 2015.

[5] N. Cho and Y. Kim, "Modified pure proportional navigation guidance law for impact time control," Journal of Guidance, Control, and Dynamics, vol. 39, no. 4, pp. 852-872, 2016.

[6] I. S. Jeon, J. I. Lee, and M. J. Tahk, "Impact-time-control guidance with generalized proportional navigation based on nonlinear formulation," Journal of Guidance, Control, and Dynamics, vol. 39, no. 8, pp. 1887-1892, 2016.

[7] D.-K. Sang and M.-J. Tahk, "Guidance law switching logic considering the seeker's field-of-view limits," Proceedings of the Institution of Mechanical Engineers, Part G: Journal of Aerospace Engineering, vol. 223, no. 8, pp. 1049-1058, 2009.

[8] Y. Zhang, X. Wang, and H. Wu, "Impact time control guidance law with field of view constraint," Aerospace Science and Technology, vol. 39, pp. 361-369, 2014.

[9] Y. Zhang, X. Wang, and H. Wu, "Impact time control guidance with field-of-view constraint accounting for uncertain system lag," Proceedings of the Institution of Mechanical Engineers, Part G: Journal of Aerospace Engineering, vol. 230, no. 3, pp. 515-529, 2016.
[10] J. Wang and R. Zhang, "Terminal guidance for a hypersonic vehicle with impact time control," Journal of Guidance, Control, and Dynamics, vol. 41, no. 8, pp. 1790-1798, 2018.

[11] N. Harl and S. N. Balakrishnan, "Impact time and angle guidance with sliding mode control," IEEE Transactions on Control Systems Technology, vol. 20, no. 6, pp. 1436-1449, 2012.

[12] S. R. Kumar and D. Ghose, "Impact time guidance for large heading errors using sliding mode control," IEEE Transactions on Aerospace and Electronic Systems, vol. 51, no. 4, pp. 31233138, 2015.

[13] D. Cho, H. J. Kim, and M. J. Tahk, "Nonsingular sliding mode guidance for impact time control," Journal of Guidance, Control, and Dynamics, vol. 39, no. 1, pp. 61-68, 2016.

[14] Y. Zhao, Y. Sheng, and X. Liu, "Analytical impact time and angle guidance via time-varying sliding mode technique," ISA Transactions, vol. 62, pp. 164-176, 2016.

[15] M. Kim, B. Jung, B. Han, S. Lee, and Y. Kim, "Lyapunov-based impact time control guidance laws against stationary targets," IEEE Transactions on Aerospace and Electronic Systems, vol. 51, no. 2, pp. 1111-1122, 2015.

[16] T. H. Kim, C. H. Lee, I. S. Jeon, and M. J. Tahk, “Augmented polynomial guidance with impact time and angle constraints," IEEE Transactions on Aerospace and Electronic Systems, vol. 49, no. 4, pp. 2806-2817, 2013.

[17] M. Snyder, Z. Qu, R. Hull, and R. Prazenica, "Quad-segment polynomial trajectory guidance for impact-time control of precision-munition strike," IEEE Transactions on Aerospace and Electronic Systems, vol. 52, no. 6, pp. 3008-3023, 2016.

[18] R. Tekin, K. S. Erer, and F. Holzapfel, "Polynomial shaping of the look angle for impact-time control," Journal of Guidance, Control, and Dynamics, vol. 40, no. 10, pp. 2668-2673, 2017.

[19] R. Tekin, K. S. Erer, and F. Holzapfel, "Control of impact time with increased robustness via feedback linearization," Journal of Guidance, Control, and Dynamics, vol. 39, no. 7, pp. 16821689, 2016.

[20] R. Tekin, K. S. Erer, and F. Holzapfel, “Adaptive Impact Time Control via Look-Angle Shaping Under Varying Velocity," Journal of Guidance, Control, and Dynamics, vol. 40, no. 12, pp. 3247-3255, 2017.

[21] R. Tekin, K. S. Erer, and F. Holzapfel, "Impact Time Control with Generalized-Polynomial Range Formulation," Journal of Guidance, Control, and Dynamics, vol. 41, no. 5, pp. 11901195, 2018.

[22] S. Kang, R. Tekin, and F. Holzapfel, "Generalized impact time and angle control via look-angle shaping," Journal of Guidance, Control, and Dynamics, vol. 42, no. 3, pp. 695-702, 2019.

[23] S. Gutman, "Impact-time vector guidance," Journal of Guidance, Control, and Dynamics, vol. 40, no. 8, pp. 2110-2114, 2017.

[24] A. Saleem and A. Ratnoo, "A Nonlinear Guidance Law for Impact Time Control," in 2015 American Control Conference (ACC), Chicago, IL, USA, 2015.

[25] I. S. Jeon and J. I. Lee, "Impact-time-control guidance law with constraints on seeker look angle," IEEE Transactions on Aerospace and Electronic Systems, vol. 53, no. 5, pp. 2621-2627, 2017.

[26] X. Chen and J. Wang, "Nonsingular sliding-mode control for field-of-view constrained impact time guidance," Journal of Guidance, Control, and Dynamics, vol. 41, no. 5, pp. 1214$1222,2018$. 
[27] X. Chen and J. Wang, "Sliding-mode guidance for simultaneous control of impact time and angle," Journal of Guidance, Control, and Dynamics, vol. 42, no. 2, pp. 394-401, 2019.

[28] Q. Hu, T. Han, and M. Xin, "Sliding-mode impact time guidance law design for various target motions," Journal of Guidance, Control, and Dynamics, vol. 42, no. 1, pp. 136-148, 2019.

[29] A. Saleem and A. Ratnoo, "Lyapunov-based guidance law for impact time control and simultaneous arrival," Journal of Guidance, Control, and Dynamics, vol. 39, no. 1, pp. 164173, 2016.

[30] K. S. Erer and R. Tekin, "Impact time and angle control based on constrained optimal solutions," Journal of Guidance, Control, and Dynamics, vol. 39, no. 10, pp. 2448-2454, 2016.

[31] S. Arita and S. Ueno, "Optimal feedback guidance for nonlinear missile model with impact time and angle constraints," in AIAA Guidance, Navigation, and Control (GNC) Conference, Boston, MA, 2013. 


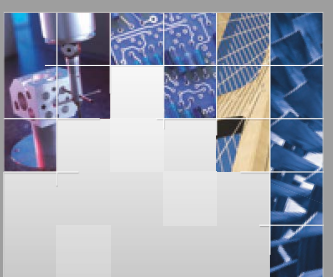

\section{Enfincering}
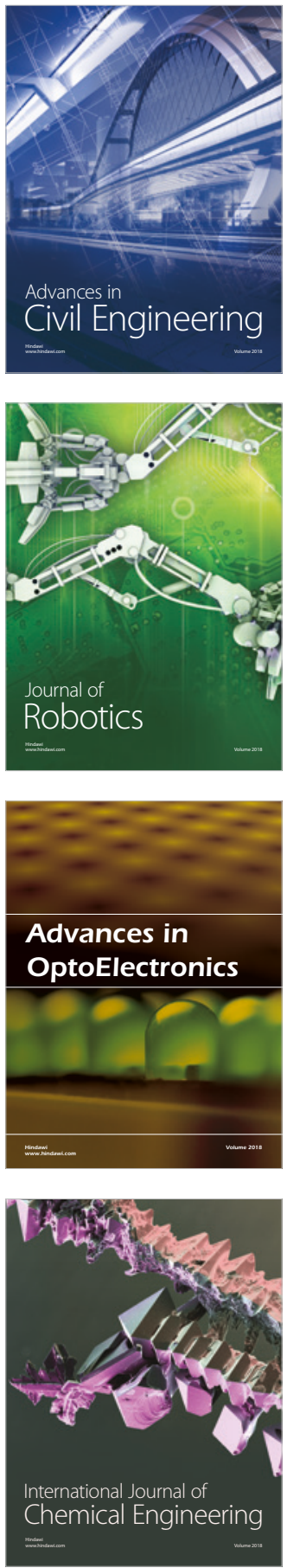

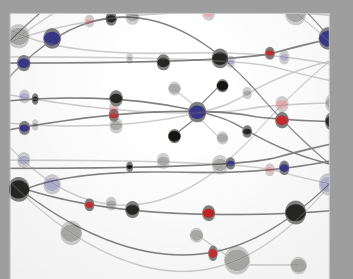

\section{Rotating \\ Machinery}

The Scientific World Journal

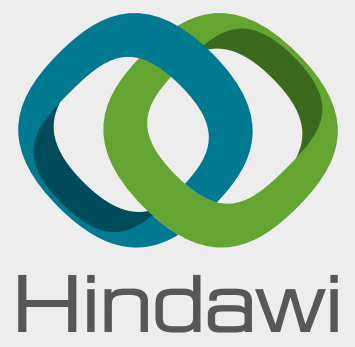

Submit your manuscripts at

www.hindawi.com
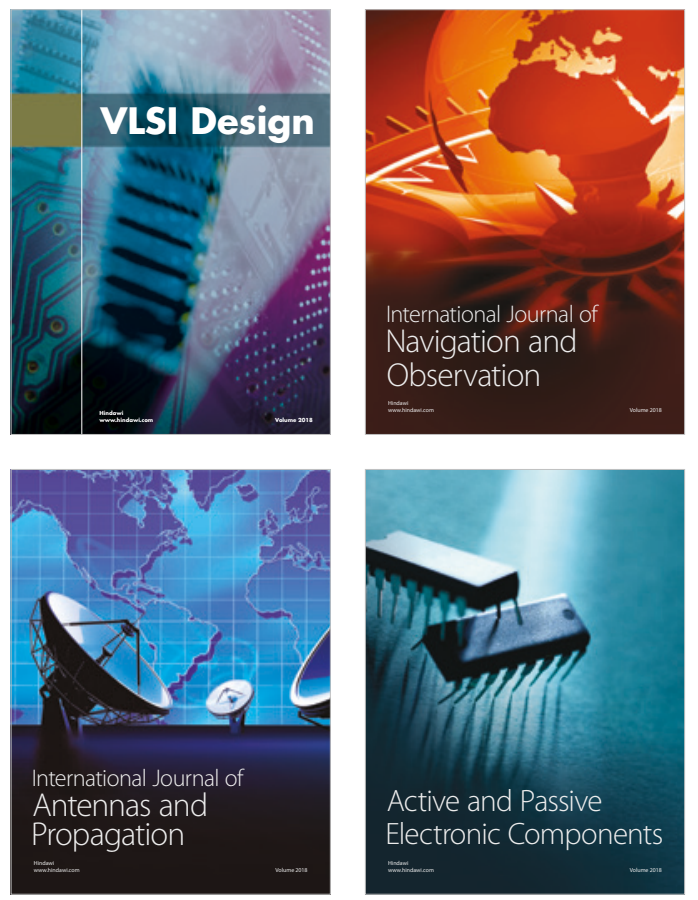
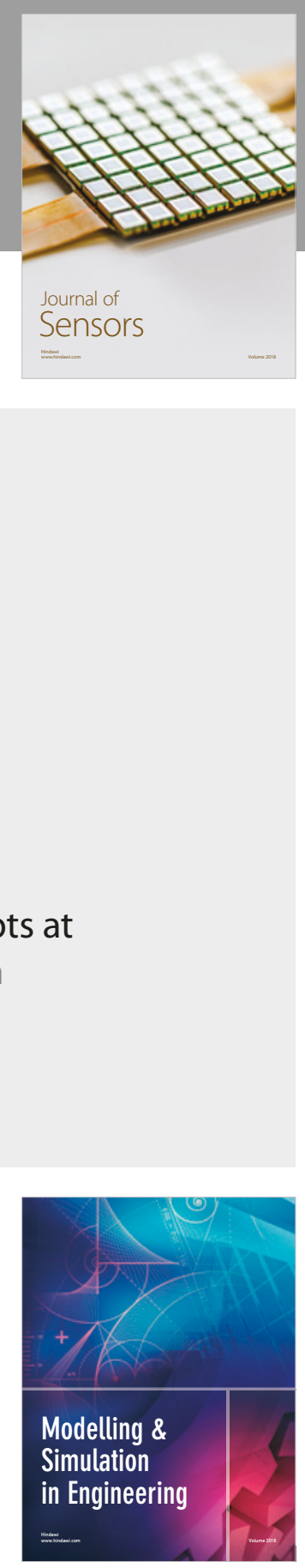

\section{Advances \\ Multimedia}
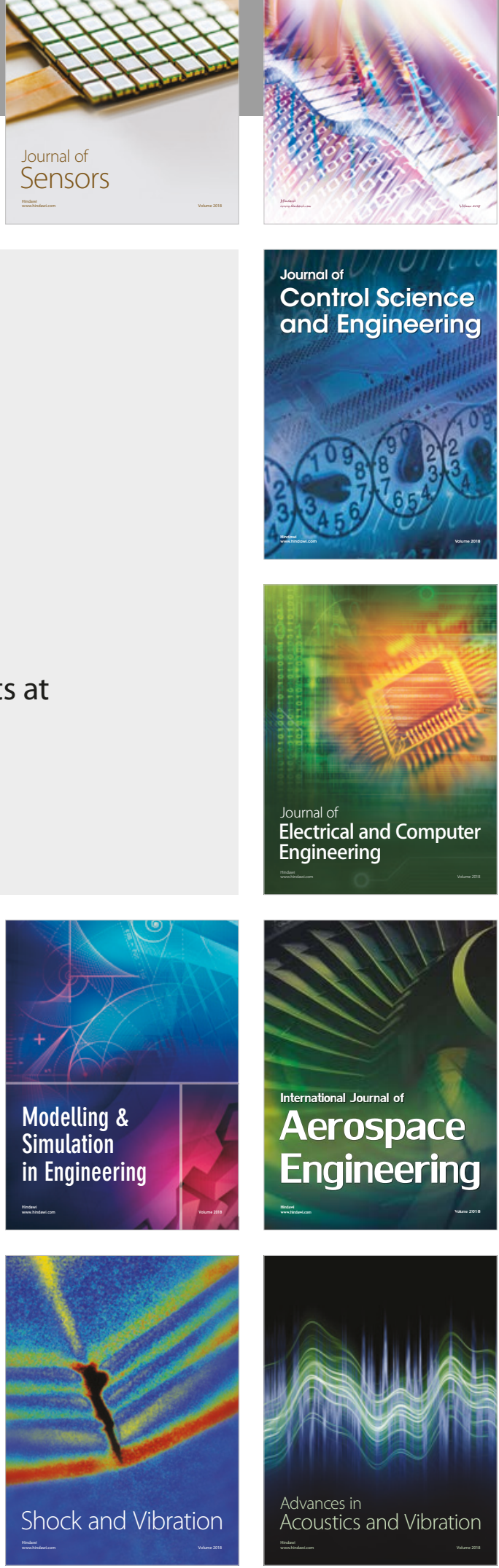